\title{
Avaliação do grau de conversão de resinas compostas fotoativadas em diferentes tempos e potências
}

\section{Evaluation of the degree of conversion of composite resins photoactivated in different curing times and power densities}

\author{
Anna Letícia Xavier de Lima* \\ Paulo Henrique de Souza** \\ Débora Michelle Gonçalves de Amorim*** \\ Sergei Godeiro Fernandes Rabelo Caldas ${ }^{* * *}$ \\ Marília Regalado Galvão ${ }^{* * * *}$
}

\section{Resumo}

Introdução: uma polimerização adequada é crucial para a obtenção das propriedades físicas ideais e o desempenho clínico das resinas compostas. Objetivo: avaliar o grau de conversão (GC) e dureza de resinas compostas fotoativadas com diferentes potências e tempos de polimerização. Materiais e método: foram confeccionados oitenta $(n=80)$ corpos de prova de resina composta (Filtek ${ }^{T M}$ Z350 XT na cor A2B) com 4 $\mathrm{mm}$ de diâmetro e $2 \mathrm{~mm}$ de espessura, desses, quarenta $(n=40)$ foram destinados à avaliação do grau de conversão e quarenta $(n=40)$ para dureza. Foram feitos dez $(n=10)$ corpos de prova para cada grupo avaliado, sendo cinco para avaliar o grau de conversão e cinco, a dureza (P10T2, P7T2, P4T2, P1T2, P10T4, P7T4, P4T4, P1T4). Tendo as resinas do grau de conversão e de dureza fotoativadas por $L E D S$ em 20 segundos na intensidade de potência de $1.000 \mathrm{~mW} / \mathrm{cm}^{2}, 700 \mathrm{~mW} / \mathrm{cm}^{2}, 400$ $\mathrm{mW} / \mathrm{cm}^{2}$ e $100 \mathrm{~mW} / \mathrm{cm}^{2}$, os grupos P10T2, P7T2, P4T2 e P1T2, respectivamente. Os grupos P10T4, P7T4, P4T4 e P1T4 foram polimerizados na mesma intensidade de potência de luz, no entanto, com tempo de 40 segundos. Resultado: em relação ao grau de conversão, as resinas do P1T2 tiveram o menor valor, alcançado em média 22,67\%, e o P10T4, com média $87,09 \%$, alcançou o maior valor. Em relação à dureza, as resinas do P1T2 tiveram também a pior média, com 30,93 Kgf/ $\mathrm{mm}^{2}$, e o P10T4, a melhor média, com 82,02 Kgf/mm². Conclusão: quanto maior a potência do aparelho foto- polimerizador, maior será o GC e a dureza das resinas compostas. Os resultados deste estudo sugerem que um aparelho com intensidade de $100 \mathrm{~mW} / \mathrm{cm}^{2}$ não oferece um grau de conversão e dureza satisfatório, comprometendo o desempenho do material restaurador.

Palavras-chave: Dureza. Polimerização. Resinas compostas.

\section{Introdução}

Desenvolvidas por Bowen durante a década de 1960, as resinas compostas à base de BisGMA passaram por transformações com o intuito de melhorar suas propriedades mecânicas e estéticas ${ }^{1}$. Concomitantemente à evolução desses materiais, os pacientes buscam cada vez mais a excelência estética ao sorrir, o que leva ao desenvolvimento de resinas que, além de devolver a forma e função do dente, favoreçam a harmonia do sorriso de maneira natural. Sendo assim, por proporcionar ao profissional a capacidade de restabelecer a estética do sorriso, confeccionar restaurações em dentes posteriores, além do seu baixo custo, quando comparadas

***** Professor doutor adjunto I, Departamento de Odontologia, Universidade Federal do Rio Grande do Norte, Natal, RN, Brasil.

Professora doutora adjunta II, Departamento de Odontologia, Universidade Federal do Rio Grande do Norte, Natal, RN, Brasil. 
aos sistemas cerâmicos, o uso de resinas compostas difundiu-se na prática odontológica mundial ${ }^{2}$.

Os pesquisadores têm grande interesse no estudo da qualidade da polimerização, pois a reação química é ativada por meio de fotoiniciadores. É de suma importância uma polimerização de qualidade para obter-se o máximo de resistência para cada material $^{3}$. Por esses motivos, novos estudos procuram encontrar a proporção adequada de densidade de potência necessária para uma eficiente conversão dos monômeros resinosos em polímeros, que trará como consequência a melhoria de suas propriedades físicas e químicas ${ }^{4}$.

O grau de conversão está relacionado à quantidade de monômero da resina composta que se converteu na cadeia polimérica, ou seja, está intimamente associado ao processo de polimerização do compósito. Uma baixa conversão do compósito pode resultar em monômeros livres, não reagentes, que podem ser dissolvidos em ambientes úmidos, resultando assim na degradação do material e comprometendo a longevidade da restauração. O grau de conversão é de grande importância para auxiliar na investigação do desempenho mecânico da resina e da biocompatibilidade, pois esse processo está diretamente relacionado à resistência à fratura, à dureza e à solubilidade do material ${ }^{5}$.

Associadas a esse fator, avaliações clínicas e laboratoriais sugerem que há associação entre o grau de conversão e as propriedades físico-mecânicas, tais como estabilidade de cor, resistência ao desgaste, à flexão ou à tração das resinas ${ }^{6}$. A densidade de potência emitida pelas fontes de luz deve ser no mínimo de $300 \mathrm{~mW} / \mathrm{cm}^{2}$ a $400 \mathrm{~mW} / \mathrm{cm}^{2}$ para polimerizar adequadamente um incremento de $1,5 \mathrm{~mm}$ a $2 \mathrm{~mm}$ de resina composta ${ }^{1,7}$. Logo, quanto mais eficiente for a unidade fotopolimerizadora, mais fótons estarão disponíveis para absorção, em consequência, mais moléculas de canforoquinona (fotoiniciador) ficarão excitadas, favorecendo assim um dos fatores necessários para uma maior polimerização ${ }^{8}$.

No início da polimerização, as moléculas de fotoiniciadores são ativadas pela luz, as quais ativam os monômeros, que, por sua vez, reagem com outros monômeros formando a cadeia polimérica. No interior da estrutura da resina polimerizada, podem existir certos monômeros presos que não reagiram, que, além de trazer problemas citotóxicos, podem se solubilizar no ambiente bucal e provocar espaços vazios na restauração e, por consequência, a ocorrência de cáries secundárias. Por essa razão, o grau de conversão (GC) é uma mensuração de reação química frequentemente testada in vitro.

Para avaliar o GC, existem vários testes, mas a técnica utilizada com mais frequência é a espectroscopia infravermelha transformada de Fourier (FT-IR). A FT-IR é utilizada para determinar o GC por intermédio da proporção de concentração de duplas ligações de carbono alifáticas antes e após a polimerização do material ${ }^{9}$. $\mathrm{O}$ percentual de du- plas ligações de carbono alifáticas não convertidas (\% $\mathrm{C}=\mathrm{C}$ ) é determinado pela taxa da intensidade de absorção entre ligações $\mathrm{C}=\mathrm{C}\left(1.638 \mathrm{~cm}^{-1}\right)$ e ligações $\mathrm{C}=\mathrm{O}\left(1.730 \mathrm{~cm}^{-1}\right)$, antes e após a polimerização. $\mathrm{O}$ grau de conversão correspondente é calculado pela subtração dessa taxa de $100 \%{ }^{6}$.

A dureza é uma propriedade que influencia diretamente a longevidade clínica de um material resinoso, pois existe uma relação direta entre essa propriedade e a resistência mecânica e a resistência ao desgaste desses materiais. Essa propriedade é medida por meio da capacidade de resistência do material à penetração por ponta dura. Frequentemente, os testes realizados para determinar a dureza dos materiais odontológicos são: Brinell, Rockwell, Vickers e Knoop, sendo que os dois primeiros testes utilizam áreas mais extensas do material para analisar a dureza; os dois últimos avaliam a microdureza, por utilizar uma pequena área do material. Dessa forma, os testes de Vickers e Knoop são os de escolha para a análise da dureza das resinas compostas $^{2}$.

O valor percentual do GC quantifica a reação química, embora não seja uma propriedade do material restaurador resinoso, estudos apontam que um baixo valor do GC afeta o desempenho clínico aceitável. Para as camadas de restaurações oclusais, valores abaixo de $55 \%$ não são aconselhados ${ }^{10}$.

Portanto, por apresentar essa correlação, é de grande importância avaliar o grau de conversão das resinas compostas, levando em consideração todas as influências possíveis e as propriedades mecânicas, como a dureza, para que, assim, sejam confeccionadas restaurações de boa qualidade e com maior tempo de vida clínica útil.

\section{Materiais e método}

Foram confeccionados, ao todo, oitenta corpos de prova de resina composta (Filtek ${ }^{\mathrm{TM}} \mathrm{Z} 350 \mathrm{XT}$ na cor A2B), quarenta corpos de prova para avaliação do grau de conversão e quarenta para avaliação da dureza. Para a confecção das amostras, foi utilizada uma matriz metálica com diâmetro interno de 4 mm e espessura de $2 \mathrm{~mm}$ (ISO 4049). Uma lamínula de vidro e a matriz metálica foram posicionadas sobre a placa de vidro. A resina foi inserida em único incremento no orifício da matriz e, em seguida, foram posicionados uma tira de poliéster e um peso metálico de $1 \mathrm{~kg}$, que continha uma abertura para adaptação e posicionamento da ponta do aparelho fotopolimerizador (Optilight LD MAX, Gnatus, Ribeirão Preto, SP, Brasil). A densidade de potência do aparelho fotopolimerizador foi aferida sempre antes de realizar a fotoativação com o aparelho radiômetro (RD-7, ECEL, Ribeirão Preto, SP, Brasil). As resinas foram fotoativadas de acordo com as recomendações do fabricante e com o grupo em questão. 
Para avaliação do grau de conversão e dureza, foram confeccionados cinco corpos de prova para cada grupo avaliado (P10T2; P7T2; P4T2; P1T2; P10T4; P7T4; P4T4; P1T4) com a resina composta e fotoativada por LEDs, totalizando oitenta corpos de prova. As resinas dos grupos P10T2, P7T2, P4T2 e P1T2 foram fotoativas por 20 segundos na intensidade de potência de $1.000 \mathrm{~mW} / \mathrm{cm}^{2}$ (P10T2), 700 $\mathrm{mW} / \mathrm{cm}^{2}$ (P7T2), $400 \mathrm{~mW} / \mathrm{cm}^{2}$ (P4T2) e $100 \mathrm{~mW} / \mathrm{cm}^{2}$ (P1T2). Os grupos P10T4, P7T4, P4T4 e P1T4 foram fotoativados na mesma ordem de intensidade de potência dos grupos anteriores, no entanto, com tempo de polimerização de 40 segundos. Após a confecção dos corpos de prova, eles foram armazenados por um período de sete dias, em recipientes plásticos escuros, a seco, em estufa à temperatura de $37^{\circ} \mathrm{C}$ $\left( \pm 1^{\circ} \mathrm{C}\right)$. Logo após esse período, para o grau de conversão, os corpos de prova foram posicionados e avaliados no espectrofotômetro (Spectrum 65 FT-IR) na faixa de $4.000 \mathrm{~cm}^{-1}$ a $300 \mathrm{~cm}^{-1}$, utilizando acessório de refletância difusa, operando com 32 scans, resolução de $4 \mathrm{~cm}^{-1}$, acoplado a um microcomputador servidor. Os espectros foram obtidos pela técnica de transmissão, observando-se os picos de absorbância. Após a obtenção dos picos de absorbância no espectrofotômetro, o percentual de duplas ligações carbônicas não convertidas $(\% \mathrm{C}=\mathrm{C})$ foi determinado pela taxa de intensidade de absorção entre ligações duplas de carbono alifáticas $\left(1.637 \mathrm{~cm}^{-1}\right)$ e ligações simples de carbono aromáticas $\left(1.610 \mathrm{~cm}^{-1}\right)$.

Para avaliação da dureza, os corpos de prova foram levados ao durômetro digital (MV 2000A) para determinar o valor médio de dureza em microvickers. Foi utilizada uma carga de 50 gramas força (gf) e tempo de 15 segundos. Para cada corpo de prova, oito medidas foram realizadas nas superfícies de topo e base para determinar um valor médio da dureza (microvickers) para cada corpo de prova.

\section{Resultado}

Para todos os grupos, valores de média e desvio padrão (DP) relativos ao grau de conversão e dureza foram obtidos a partir de cinco corpos de prova. Os resultados de conversão foram comparados por meio de análise de variância (ANOVA) a dois fatores (variáveis tempo e intensidade) e pós-teste de Tukey, ao nível de significância de $5 \%$.

De acordo com os dados da Tabela 1, na polimerização da resina nas potências $1.000 \mathrm{~mW} / \mathrm{cm}^{2} \mathrm{e}$ $700 \mathrm{~mW} / \mathrm{cm}^{2}$, tanto nos tempos de 20 quanto de 40 segundos, o grau de conversão não aumenta significativamente. Diferenças estatísticas significantes foram encontradas ao verificar que o GC sempre foi maior naquelas resinas que foram fotoativadas nas maiores intensidades de potência.
Tabela 1 - Valores médios do grau de conversão (\%), desvio padrão (DP) e $p\left(^{*}\right)$

\begin{tabular}{|l|c|c|}
\hline $\begin{array}{c}\text { Potência } \\
\left(\mathrm{mW} / \mathrm{cm}^{2}\right)\end{array}$ & $\begin{array}{c}\text { Tempo (20 s) } \\
\text { Média (DP) }\end{array}$ & $\begin{array}{c}\text { Tempo (40 s) } \\
\text { Média (DP) }\end{array}$ \\
\hline 1.000 & $85,24(1,42) \mathrm{aA}$ & $87,09(1,80) \mathrm{aA}$ \\
700 & $81,07(2,40) \mathrm{aA}$ & $82,75(2,59) \mathrm{aA}$ \\
400 & $62,54(2,04) \mathrm{aB}$ & $67,88(2,20) \mathrm{bB}$ \\
100 & $22,67(3,71) \mathrm{aC}$ & $26,42(2,34) \mathrm{aC}$ \\
\hline
\end{tabular}

Fonte: dos autores.

* Letras diferentes (letras maiúsculas na mesma coluna e letras minúsculas na mesma linha) indicam diferença estatisticamente significativa $(p>0,05)$.

Os grupos P1T2 e P1T4 não apresentaram diferenças estatisticamente significantes, evidenciando que mesmo com o dobro do tempo de exposição à luz em menor intensidade não houve melhora nos resultados. Os valores de dureza obtidos foram comparados em análise de variância (ANOVA 2-way) e pós-teste de Tukey, ao nível de significância de $5 \%$.

Quanto à influência da potência do aparelho fotoativador, verificou-se que nas resinas nas quais se aplicou potência de $100 \mathrm{~mW} / \mathrm{cm}^{2}$ houve um comprometimento significativo na microdureza do material (Tabela 2).

Tabela 2 - Valores médios de microdureza $\left(\mathrm{Kgf} / \mathrm{mm}^{2}\right)$ e desvio padrão (DP)

\begin{tabular}{|c|c|c|}
\hline $\begin{array}{c}\text { Potência } \\
\left(\mathrm{mW} / \mathrm{cm}^{2}\right)\end{array}$ & $\begin{array}{c}\text { Tempo (20s) } \\
\text { Média (DP) }\end{array}$ & $\begin{array}{c}\text { Tempo (40s) } \\
\text { Média (DP) }\end{array}$ \\
\hline 1.000 & $69,74(1,70) \mathrm{aA}$ & $82,02(1,86) \mathrm{bA}$ \\
700 & $68,98(3,43) \mathrm{aA}$ & $75,09(5,30) \mathrm{aB}$ \\
400 & $64,02(2,68) \mathrm{aA}$ & $64,64(3,48) \mathrm{aC}$ \\
100 & $30,93(6,69) \mathrm{aB}$ & $51,63(4,49) \mathrm{bC}$ \\
\hline
\end{tabular}

Fonte: dos autores.

* Letras diferentes (Letras maiúsculas na mesma coluna e Letras minúsculas na mesma linha) indicam diferença estatisticamente significativa $(p>0,05)$.

\section{Discussão}

As resinas compostas apresentam-se inicialmente em uma fase plástica, por meio da luz visível, emitida por aparelhos, ocorre sua polimerização, que deve ser de elevado grau para poder proporcionar as propriedades físico-mecânicas desejadas e minimizar os riscos de toxicidade ao complexo dentina-polpa ${ }^{9}$.

A densidade de potência emitida pelas fontes de luz deve ser no mínimo de $300 \mathrm{~mW} / \mathrm{cm}^{2}$ a 400 $\mathrm{mW} / \mathrm{cm}^{2}$ para polimerizar adequadamente um incremento de $1,5 \mathrm{~mm}$ a $2 \mathrm{~mm}$ de resina composta ${ }^{7,11}$. Se a restauração de resina não recebe a densidade de potência adequada, pode ocorrer acúmulo de monômero residual, levando a um baixo GC, aumento da citotoxicidade, redução da dureza, diminuição no módulo de elasticidade, aumento do desgaste e ruptura das margens da restauração ${ }^{12}$. 
O GC final depende tanto de fatores intrínsecos ao material quanto de fatores extrínsecos. Os intrínsecos seriam a estrutura química do monômero e a concentração de fotoiniciadores, já os fatores extrínsecos estariam relacionados às condições de fotopolimerização. $\mathrm{O}$ valor percentual do GC não foi ainda estabelecido para o desempenho clínico aceitável, porém, de acordo com alguns autores, para as camadas de restaurações oclusais, valores abaixo de $55 \%$ não são aconselhados ${ }^{10}$. Corroborando com essa posição, este estudo mostrou que as resinas fotoativadas a partir da intensidade de potência de 400 $\mathrm{mW} / \mathrm{cm}^{2}$ tiveram a média de $62,54 \%$, para aquelas polimerizadas por 20 segundos, e $67,88 \%$, para as polimerizadas por 40 segundos. Ou seja, tiveram uma porcentagem do GC suficiente para ser considerada aconselhável. Os valores foram ainda maiores naquelas resinas fotoativadas com maiores intensidades de potência, tendo o GC em mais de $80 \%$ naquelas com intensidade de potência entre $700 \mathrm{~mW} /$ $\mathrm{cm}^{2}$ e $1.000 \mathrm{~mW} / \mathrm{cm}^{2}$. Já as resinas fotoativadas com intensidades de $100 \mathrm{~mW} / \mathrm{cm}^{2}$ tiveram uma porcentagem de GC muito baixa, independente do tempo de polimerização, sendo $22,67 \%$ para as fotoativadas por 20 segundos e $26,42 \%$ para as fotoativadas por 40 segundos, não sendo, dessa forma, aconselhada essa intensidade de luz para uso clínico.

Foi possível observar que as resinas compostas fotoativadas com aparelhos de maior intensidade de potência apresentaram maior GC, independente do tempo. O que corrobora os estudos de alguns autores que relatam que quanto mais eficiente for a unidade fotopolimerizadora, mais fótons estarão disponíveis para absorção e, em consequência, mais moléculas do fotoiniciador que estão na resina ficarão excitadas, promovendo maior polimerização e, consequentemente, maior GC $^{5}$. Essa avaliação concorda também com os estudos de alguns autores que mostraram que o GC era maior nas resinas fotoativadas com LEDs de alta intensidade ${ }^{13}$.

Apesar de os fabricantes recomendarem o tempo de polimerização de 20 segundos, foi observado que o GC foi maior em todos os grupos em que o tempo de polimerização foi de 40 segundos, embora não houvesse diferença estatisticamente significativa. Como o GC não teve aumento estatisticamente significante, isso pode ser explicado, pois, durante a progressão da reação de polimerização, a taxa de polimerização diminui rapidamente à medida que a rigidez do material impede a mobilidade dos monômeros remanescentes e das cadeias poliméricas em crescimento para continuar a reação, mesmo com o aumento da densidade total de energia ${ }^{14}$.

A partir do exposto, fica evidente que o GC é influenciado diretamente pela intensidade de potência dos aparelhos fotopolimerizadores e que os maiores valores de GC estão associados às mais altas potências. No entanto, não ficou tão clara a evidência de que o aumento no tempo de fotoativação leva ao aumento no GC, visto que os valores encontrados não tiveram significância estatística.

Outros estudos relatam que regiões com concentração mais elevada do GC tiveram como consequência maior dureza superficial local. Sugerindo, assim, que maior exposição à luz pode aumentar o nível de valores de dureza, mas não afeta a distribuição de dureza. Uma distribuição homogênea da dureza não pode ser alcançada simplesmente por aumentar o tempo de exposição à luz. Esse fato pode ser explicado considerando-se que com o aumento de GC, consequentemente, temos uma maior estrutura básica de polímeros formados ${ }^{15}$.

Entre as medidas mais importantes para se comparar materiais restauradores estão os valores de dureza em Vickers, uma propriedade mecânica que deve ser levada em consideração, especialmente, quando são confrontados com grandes áreas de esforço mastigatório ${ }^{16,17}$.

Segundo alguns autores, ainda não há consenso sobre um valor de dureza ideal para resinas compos$\operatorname{tas}^{18}$. Entretanto, um estudo demonstrou que, para resinas compostas, o valor considerado correto seria um valor de dureza superior a 50 (VHN - número de dureza em Vickers ${ }^{18}$. Corroborando esses autores, este estudo mostrou que as resinas fotoativadas com intensidade de potência de $1.000 \mathrm{~mW} / \mathrm{cm}^{2}$ (G1), $700 \mathrm{~mW} / \mathrm{cm}^{2}$ (G2) e $400 \mathrm{~mW} / \mathrm{cm}^{2}$ (G3) apresentaram valores de dureza acima de 50 VHN. Em média, o G1 apresentou uma dureza de 69,74 Vickers, o G2 68,98 Vickers e o G3 64,02 Vickers. Apenas as resinas do G4, fotoativadas com intensidade de potência de $100 \mathrm{~mW} / \mathrm{cm}^{2}$, apresentaram um valor de dureza inferior, com média de 38,47 Vickers.

\section{Conclusão}

Este estudo demonstrou que a intensidade da luz e o tempo de fotopolimerização influenciaram o grau de conversão e dureza da resina composta, evidenciando que o tempo de fotopolimerização e as potências de luz podem ter impacto direto sobre a densidade de potência, tendo grande relevância sobre as propriedades físicas, biológicas e mecânicas dos compósitos.

Os resultados do estudo sugerem que quanto maior é a potência do aparelho fotopolimerizador, maior será o grau de conversão e dureza das resinas compostas. Por fim, um aparelho fotoativador com intensidade de $100 \mathrm{~mW} / \mathrm{cm}^{2}$ não oferece um grau de conversão e microdureza satisfatório, comprometendo o desempenho clínico do material restaurador. 


\section{Abstract}

Introduction: Adequate polymerization is a crucial factor in obtaining optimal physical properties and clinical performance of composite resins. Objective: To evaluate the degree of conversion (DC) and hardness of composite resins photoactivated in different power densities and curing times. Materials and method: Eighty $(n=80)$ composite resin specimens (Filtek ${ }^{T M}$ Z350 XT color A2B) with $4 \mathrm{~mm}$ of diameter and $2 \mathrm{~mm}$ of thickness were produced, whereas forty $(n=40)$ were meant for the evaluation of degree of conversion and forty $(n=40)$ for hardness. Ten $(n=10)$ specimens were produced for each group assessed, whereas five were meant for the evaluation of the degree of conversion and five for hardness (P10T2; P7T2; P4T2; P1T2; P10T4; P7T4; P4T4; $P 1 T 4)$. Resins of the degree of conversion and hardness were photoactivated by LED for 20 seconds at power intensities of $1000 \mathrm{~mW} / \mathrm{cm}^{2}, 700 \mathrm{~mW} / \mathrm{cm}^{2}, 400 \mathrm{~mW} / \mathrm{cm}^{2}$, and $100 \mathrm{~mW} / \mathrm{cm}^{2}$, for groups P10T2, P7T2, P4T2, and P1T2, respectively. Groups P10T4, P7T4, P4T4, and P1T4 were cured in the same power intensity, but for 40 seconds. Result: Regarding the degree of conversion, P1T2 resins had the lowest mean value of $22.67 \%$, and P10T4 resins had the highest mean value of $87.9 \%$. As for hardness, P1T2 resins also had the lowest mean of $30.93 \mathrm{kgf} / \mathrm{mm}^{2}$, and P10T4 had the highest mean of $82.02 \mathrm{kgf} / \mathrm{mm}^{2}$. Conclusion: The higher the power of the curing unit, the higher the DC and hardness of composites resins. The results of this study suggest that a device with intensity of $100 \mathrm{~mW} / \mathrm{cm}^{2}$ does not provide satisfactory degree of conversion and hardness, compromising the performance of the restorative material.

Keywords: Hardness. Polymerization. Composite resins.

\section{Referências}

1. Mitra SB, Wu D, Holmes BN. An application of nanotechnology in advanced dental materials. J Am Dent Assoc 2003; 134:1382-90.

2. Souza ROA, Michida SMA, Zogheib LV, Lombardo GHL, Pereira PC, Barca DC, et al. Avaliação da dureza Vickers de resinas compostas de uso direto e indireto. Cienc Odontol Bras 2009; 12(1):23-30.

3. Ccahuana Vásquez RA, Torres CRG, Araújo MAM, Anido AA. Influência do tipo de ponteira condutora de luz de aparelhos LED na microdureza das resinas compostas. Rev Odontol Unesp 2004; 33(2):69-73.

4. Jandt KD, Mills RW, Blackwell GB, Ashworth SH. Depth of cure and compressive strength of dental composites cured with blue light emitting diodes (LEDs). Dental Materials 2000; 16(1):41-7.

5. Rastelli ANS, Jacomassi DP, Bagnato VS. Effect of power densities and irradiation times on the degree of conversion and temperature increase of a microhybrid dental composite resin. Laser PhyS 2008; 18:1074-9.

6. Neves AD, Discacciati JAC, Oréfice RL, Jansen WC. Correlação entre grau de conversão, microdureza e conteúdo inorgânico em compósitos. Pesq Odontol Bras 2002; 16(4):349-54.

7. Della Bona A, Rosa V, Cecchetti D. Influence of shade and irradiation time on the hardness of composite resins. Braz Dent J 2007; 18(3):231-4.
8. Kurachi C, Tuboy AM, Magalhaes DV, Bagnato VS. Hardness evaluation of a dental composite polymerized with experimental LED-based devices. Dent Mater 2001; 17:309-15.

9. Marovic D, Panduric V, Tarle Z, Ristic M, Sairiri K, Demoli $\mathrm{N}$, et al. Degree of conversion and microhardness of dental composite resin materials. J Molecular Structure 2013; 1044:299-302.

10. Alshali RZ, Silkikas N, Satterthwaite JD. Degree of conversion of bulk-fill compared to conventional resin-composites at two time intervals. Dental Materials 2013; 29(9):213-7.

11. Galvão MR, Costa SXS, Victorino KR, Ribeiro AA, Menezes $\mathrm{FCH}$, Rastelli ANS, et al. Influence of light guide tip used in the photo-activation on degree of conversion and hardness of one nanofilled dental composite. Laser Physics 2010; 20(12):2050-5.

12. Emami N, Söderholm KJM. How light irradiance and curing time affect monomer conversion in light-cured resin composites. Eur J of Oral Sciences 2003; 111(6):536-42.

13. Gaglianone LA, Lima AF, Araújo LSN, Cavalcanti NA, Marchi GM. Influence of different shades and LED irradiance on the degree of conversion of composite resins. Braz Oral Research 2012; 26(2):165-9.

14. Calheiros FC, Braga RR, Kawano Y, Ballester RY. Relationship between contraction stress and degree of conversion in restorative composites. Dent Mater 2004; 20(10):939-46.

15. Haenel T, Hausnerová B, Steinhaus J, Price RBT, Sullivan B, Moeginger B. Effect of the irradiance distribution from light curing units on the local micro-hardness of the surface of dental resins. Dent Mater 2015; 31:93-104.

16. Vandewalle KS, Ferracane JL, Hilton TJ, Erickson RL, Sakaguchi RL. Effect of energy density on properties and marginal integrity of posterior resin composite restorations. Dent Mater 2004; 20:96-106.

17. Craig RG, Powers JM. Restorative dental materials. 11. ed. Mosby: St. Louis; 2002.

18. Sharkey S, Ray N, Burke F, Ziada H, Hannigan A. Surface hardness of light-activated resin composites cured by two different visible-light sources: an in vitro study. Quint Int $2001 ; 32: 401-5$.

\section{Endereço para correspondência:}

Marília Regalado Galvão

Rua Dr. Múcio Galvão, 450, Bairro Vermelho

59022-530 Natal, RN - Brasil

Telefone: (84) 99650-7499

E-mail: mariliaregalado@hotmail.com

Recebido: 23/06/2016. Aceito: 22/09/2016. 